\title{
RESEARCH
}

Open Access

\section{Chest CT manifestations with emphasis on the role of CT scoring and serum ferritin/ lactate dehydrogenase in prognosis of coronavirus disease 2019 (COVID-19)}

Rehab Abdel Rahman El Bakry ${ }^{1 *}$ (D) and Ayman Ibrahim Tharwat Sayed ${ }^{2}$

\begin{abstract}
Background: In March 2020, the World Health Organization announced coronavirus disease 2019 (COVID-19) a pandemic, and because of the primary pulmonary manifestations of the disease, chest $C T$ is essential in the evaluation of those patients. The aim of the study was to evaluate the role of chest $\mathrm{CT}$ findings and chest $\mathrm{CT}$ scoring along with serum ferritin and LDH in the prognosis of COVID-19 patients in a cohort of the Egyptian population.
\end{abstract}

Results: This retrospective study included 250 patients with positive RT-PCR for COVID-19, 138 males [55.2\%] and 112 females [44.8\%], age range 17-82 years with median 49.5. Two hundred patients had a positive significant correlation between age, serum ferritin, serum $\mathrm{LDH}$, and $\mathrm{CT}$ score. Bilateral affection was $88 \%$ while unilaterality was 12\%, and peripheral chest CT findings were stratified as follows: mild [score from 1 to 10], 114 patients [57\%]; moderate [score from 11 to 19], 65 patients [32.5\%]; and severe [score from 20 to 25], 21 patients [10.5\%]. In severe cases, males constitute $85.7 \%$ while females were only $14.3 \%$. Statistical and central distribution was $67 \%$, peripheral was $31 \%$, and central was $2 \%$. Ground glass opacity (GGO) was the highest pattern 39.2\%, consolidation 31.2\%, fibrosis $15.2 \%$, and CP 13.7\%, with lymph nodes only $0.6 \%$. Fifteen cases [6\%] were critical; all showed severe scores ranging from 21 to 23 with three times increase in serum ferritin and four times increase in LDH. A follow-up study done to 8 cases [3.2\%] showed an increase in CT scoring, serum ferritin, and serum LDH.

Conclusion: Chest CT findings are crucial for early diagnosis of COVID-19 disease especially for asymptomatic patients with old age and male sex considered risk factors for poor prognosis. Chest CT score, serum ferritin, and serum LDH help in predicting the short-term outcome of the patients aiming to decrease both morbidity and mortality.

Keywords: Coronavirus, COVID-19, Chest CT, Ferritin, LDH

\footnotetext{
* Correspondence: Rehabakry_74@yahoo.com

'Department of Radiodiagnosis, Faculty of Medicine, Ain Shams University,

Cairo, Egypt

Full list of author information is available at the end of the article
}

Springer Open (c) The Author(s). 2021 Open Access This article is licensed under a Creative Commons Attribution 4.0 International License, which permits use, sharing, adaptation, distribution and reproduction in any medium or format, as long as you give appropriate credit to the original author(s) and the source, provide a link to the Creative Commons licence, and indicate if changes were made. The images or other third party material in this article are included in the article's Creative Commons licence, unless indicated otherwise in a credit line to the material. If material is not included in the article's Creative Commons licence and your intended use is not permitted by statutory regulation or exceeds the permitted use, you will need to obtain permission directly from the copyright holder. To view a copy of this licence, visit http://creativecommons.org/licenses/by/4.0/. 


\section{Background}

A viral pneumonia of unknown etiology appeared in China in late December 2019, and the Chinese scientific centers declared that the cause is a new strain of coronavirus named severe acute respiratory syndrome coronavirus 2 (SARS-CoV-2), and the disease is named coronavirus disease 2019 (COVID-19) [1]. It is a highly contagious disease, and by March 2020, the World Health Organization declared it as a pandemic causing a global health emergency [2] making early diagnosis mandatory for proper management of the patients and rapid detection of contacts for quarantine purpose; real-time reverse transcriptasepolymerase chain reaction of the viral nucleic acid (RT-PCR) within body specimens mainly nasopharyngeal or oro-phangeal swab is the gold standard for diagnosis [3]. Many publications appeared mainly focusing on the diagnosis of the disease either comparing chest CT findings with RT-PCR results [4-6] or describing the disease imaging features mainly chest CT [7-9] with paucity of articles investigating the role of chest $\mathrm{CT}$ in disease prognosis regarding helping the physician to speed up the diagnostic work flow by properly selecting patients in need of intensive medical care, or only hospital ward care or just home quarantine. The purpose of the study was to investigate the role of chest $\mathrm{CT}$ findings and $\mathrm{CT}$ scores along with serum biomarkers namely serum ferritin and lactate dehydrogenase (LDH) in the proper effective management of COVID-19 patients.

\section{Methods}

This retrospective study was done in the university hospital which included 250 patients [138 males $55.2 \%, 112$ females $44.8 \%$, age range $17-82$ years, median is 49.5 ] from first of April till 15 June 2020. The study was approved by the hospital's ethical committee. Inclusion criteria included patients with positive RT-PCR for COVID-19 in the nasopharyngeal or oro-phangeal swab; either having symptoms of viral pneumonia namely fever, cough, dyspnea, chest pain, or gastrointestinal symptoms mainly diarrhea and abdominal pain; or asymptomatic but with a history of contact to a positive COVID-19 patient. Exclusion criteria included any patient with chronic lung disease.

\section{CT scanning protocol}

All the patients underwent non-enhanced chest CT examination using either 16 or 128 multislice CT scanner. The scan was taken with the patient in the supine position, head first, from above the first rib till below the diaphragm during breath holding at end of inspiration with the following parameters: tube voltage $120 \mathrm{kV}$, adaptive tube current, pitch [0.3-1.5] with slice thickness and interslice gap both of $5 \mathrm{~mm}, 0$ gantry tilt, and FOV depending on the size of the patient. Images were reconstructed in both coronal and sagittal planes and were sent to a compatible PACS system.

CT images were evaluated by two experienced radiologists [more than 10 years of experience] independently and any disagreement was resolved by discussion. Axial images were reviewed along with the coronal and sagittal reformatted images on both lung window [window width $1500 \mathrm{HU}$, window level $700 \mathrm{HU}$ ] and mediastinal window [window width $350 \mathrm{HU}$, window level $40 \mathrm{HU}]$ settings.

\section{Image analysis}

CT images were evaluated for the following features: pulmonary manifestation localization either involving one lung or both lungs; the distribution either peripheral/subpleural, central/broncho-vascular, or both; and lesion pattern that was described in line with the Fleischner Society glossary of terms for thoracic imaging (2008) [10] including ground glass opacity [GGO] which is an area of increased attenuation not obscuring the underlying vasculature, consolidation which is an area of increased attenuation obscuring underlying pulmonary vessels, crazy paving [CP] which is an area of ground glassing with interlobular septal thickening, or mediastinal or hilar lymph nodes [with short-axis diameter more than $10 \mathrm{~mm}$ ]. Each patient was given a severity score using a semi-quantitative method for each lobe involvement as follows: 0 , no involvement; $1,<5 \%$ involvement; 2, 5-25\% involvement; 3, 26-50\% involvement; 4, 51-75\% involvement; and 5, >75\% involvement. The total CT score was the sum of each individual lobar score and it ranges from 0 to 25 [11]; then a severity grading was given to each patient as follows: mild if score is from 1 to 10 , moderate from 11 to 19 , and severe from 20 to 25 .

\section{Laboratory markers}

For each patient, serum ferritin and serum LDH were measured within 1 day of $\mathrm{CT}$.

\section{Follow-up}

A follow-up chest CT was done to 8 patients in time interval ranging from 3 to 10 days from the first scan along with serum ferritin and serum LDH due to deterioration of the clinical condition.

\section{Data collection}

Epidemiological data, laboratory results, and CT results were obtained from the hospital information system [HIS] and image storage and transmission system [PACS]. 
Table 1 Comparison between clinical picture and chest CT findings

\begin{tabular}{llll}
\hline & Positive chest CT findings & Negative chest CT findings & Total \\
\hline Symptoms & $163 / 185,88.2 \%$ & $22 / 185,11.8 \%$ & $185,74 \%$ \\
No symptoms & $37 / 65,56.9 \%$ & $28 / 65,43.1 \%$ & $65,26 \%$ \\
Total & 200 & 50 & 250 \\
\hline
\end{tabular}

\section{Statistical analysis}

IBM SPSS statistics (V. 26.0, IBM Corp., USA, 2019) was used for data analysis. Data were expressed as median and percentiles for quantitative non-parametric measures and both number and percentage for categorized data.

\section{Results}

Two hundred and fifty patients were enrolled in this study of which 138 were males [55.2\%] and 112 females [44.8\%], age range from 17 to 82 years with median 49.5 . One hundred eighteen patients [47.2\%] had underlying comorbidities as follows: forty-two [35.5\%] having hypertension, 35 [29.6\%] diabetes mellitus, 16 [13.5\%] cardiac disease, 13 [11\%] liver cirrhosis, 10 [8.4\%] renal impairment, and 2 [1.7\%] malignancy, one case with multiple myeloma and the other had colonic carcinoma.

A non-contrast chest CT scan was done to all patients within 1 week from the onset of symptoms or from the appearance of positive RT-PCR result, and estimation of serum ferritin level and serum LDH was done within 1 day of chest CT.
One hundred eighty-five patients [74\%] were symptomatizing: 163/185 patients [88.2\%] were having positive chest CT, while $22 / 185$ patients [11.8\%] had negative chest CT. Sixty-five patients [26\%] were asymptomatic: $37 / 65$ patients [56.9\%] had positive chest CT whereas 28/65 patients [43.1\%] showed no abnormality (Table 1 ).

Two hundred patients had positive chest CT findings and they were stratified as follows: mild [score from 1 to 10], 114 patients representing 57\% (Figs. 1, 2, 3, and 4); moderate [score from 11 to 19], 65 patients [32.5\%] (Figs. 5, 6, 7, 8, and 9); and severe [score from 20 to 25], 21 patients [10.5\%] (Fig. 10).

Comparison between sex and different CT stages showed no difference in the mild stage, but in the moderate stage, females were $35.4 \%$ compared to $64.6 \%$ for males and in the severe stage, females were only $14.3 \%$ compared to $85.7 \%$ for males (Table 2); also, a comparison between age and CT scoring showed that there was a statistically significant correlation between the two factors using the KruskalWallis test (Tables 3 and 4).
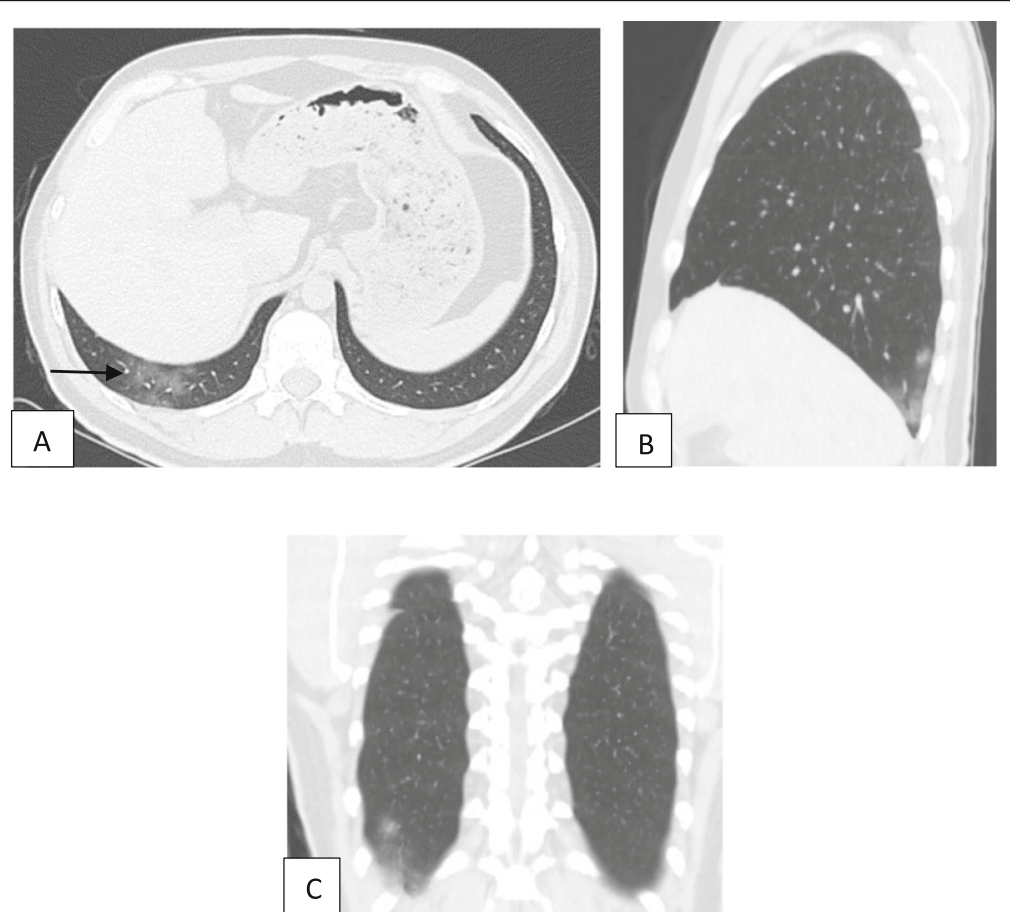

Fig. 1 Unenhanced chest CT of a 38-year-old male patient with COVID-19. a An axial lung window image shows the right peripheral subpleural area of ground glass opacity [arrow]. $\mathbf{b}$ Same finding on sagittal and $\mathbf{c}$ coronal reformatted images. CT score is 1 


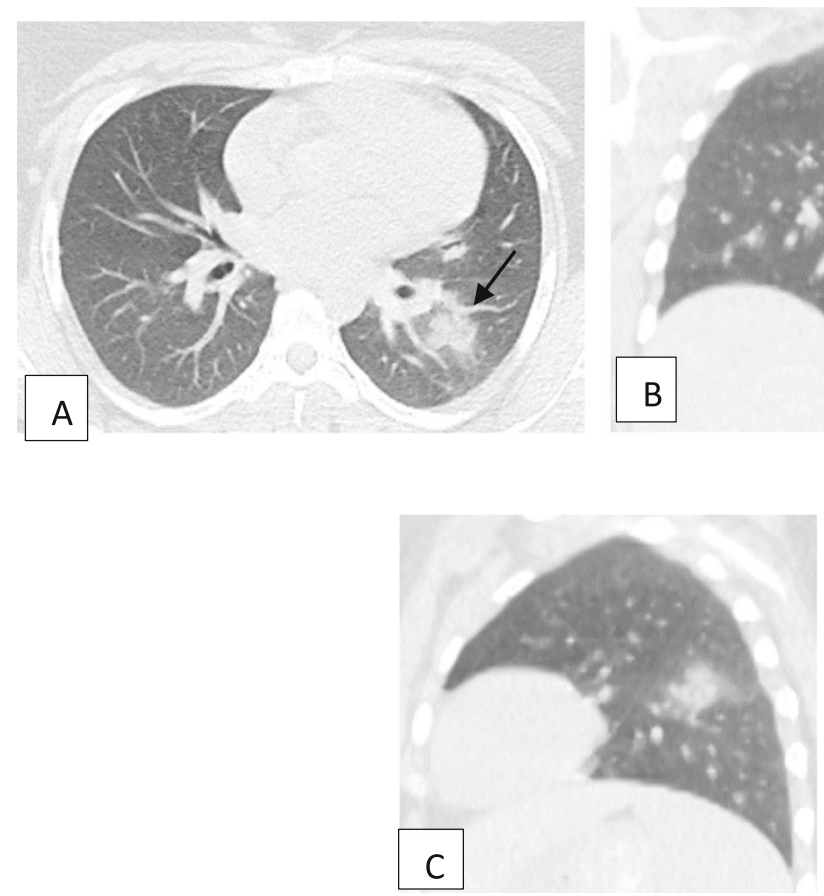

Fig. 2 Unenhanced chest CT of a 29-year-old female patient with COVID-19. a An axial lung window image shows left lower lobe central broncho-vascular patch of consolidation [arrow]. b Same finding on the coronal and c sagittal reformatted images. CT score is 2

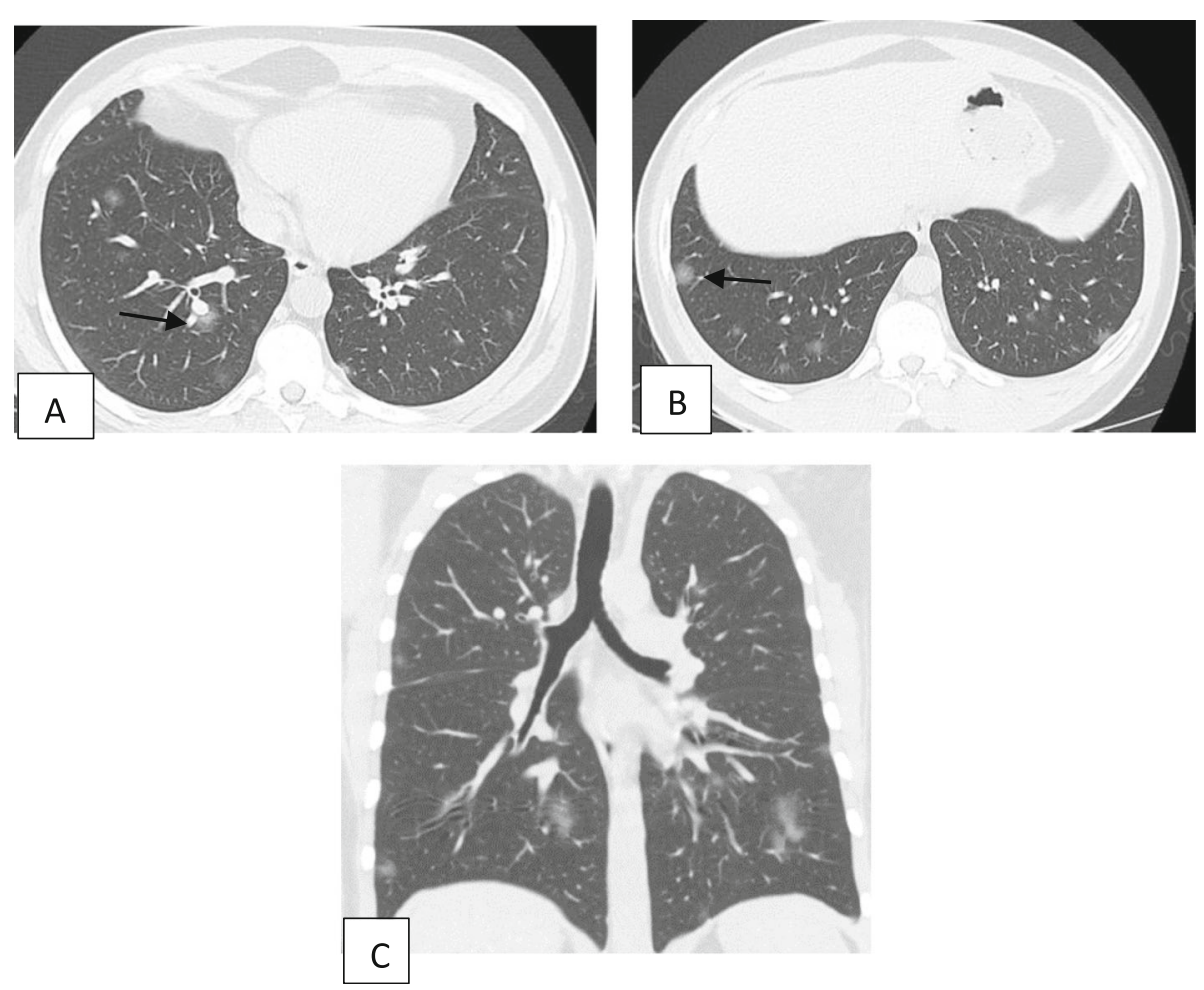

Fig. 3 Unenhanced chest CT of a 33-year-old male patient with COVID-19. a, b Axial lung window images show bilateral subpleural and broncho-vascular areas of ground glassing opacity [arrows]. c Coronal reformatted images. CT score is 5 

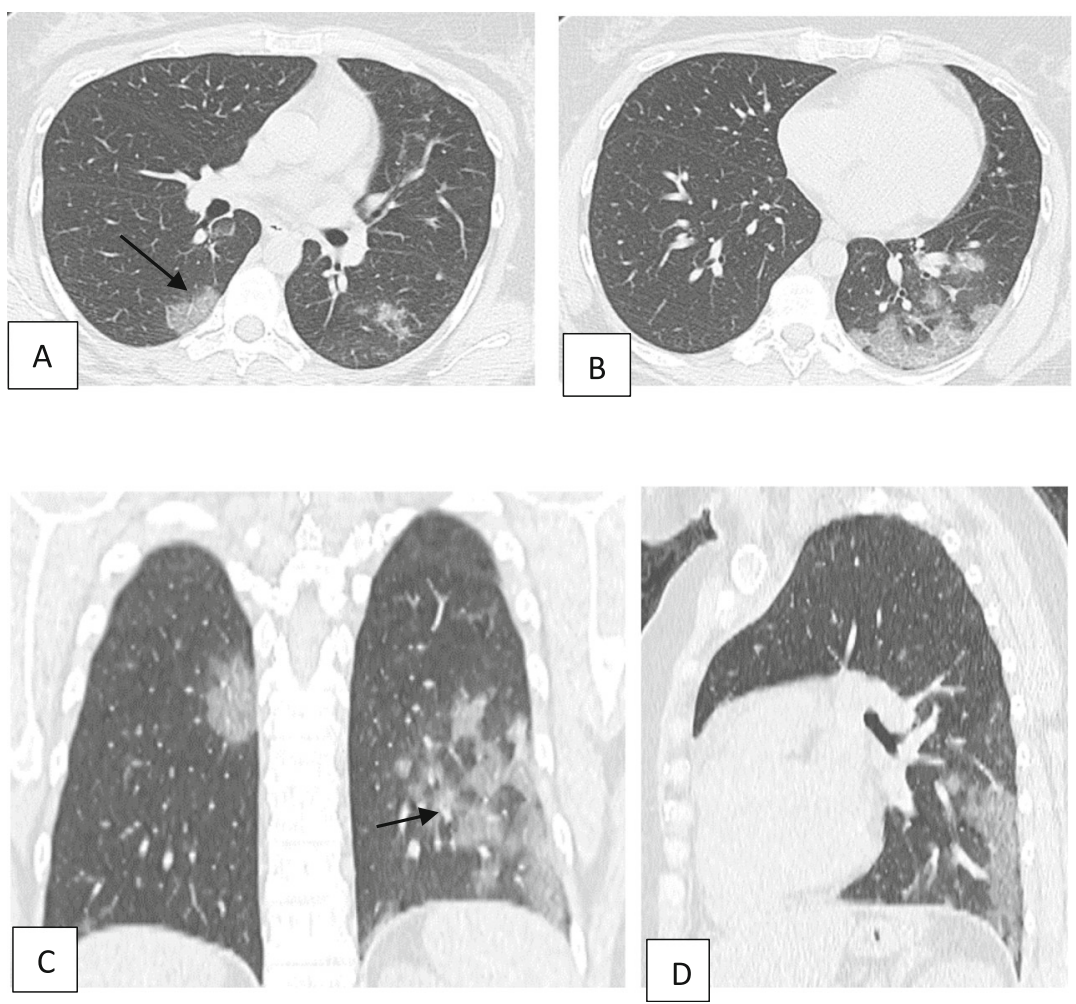

Fig. 4 Unenhanced chest CT of a 32-year-old female patient with COVID-19. a, b Axial lung window images show bilateral subpleural and broncho-vascular areas of crazy paving [arrows]. c Coronal and $\mathbf{d}$ sagittal reformatted images. CT score is 7
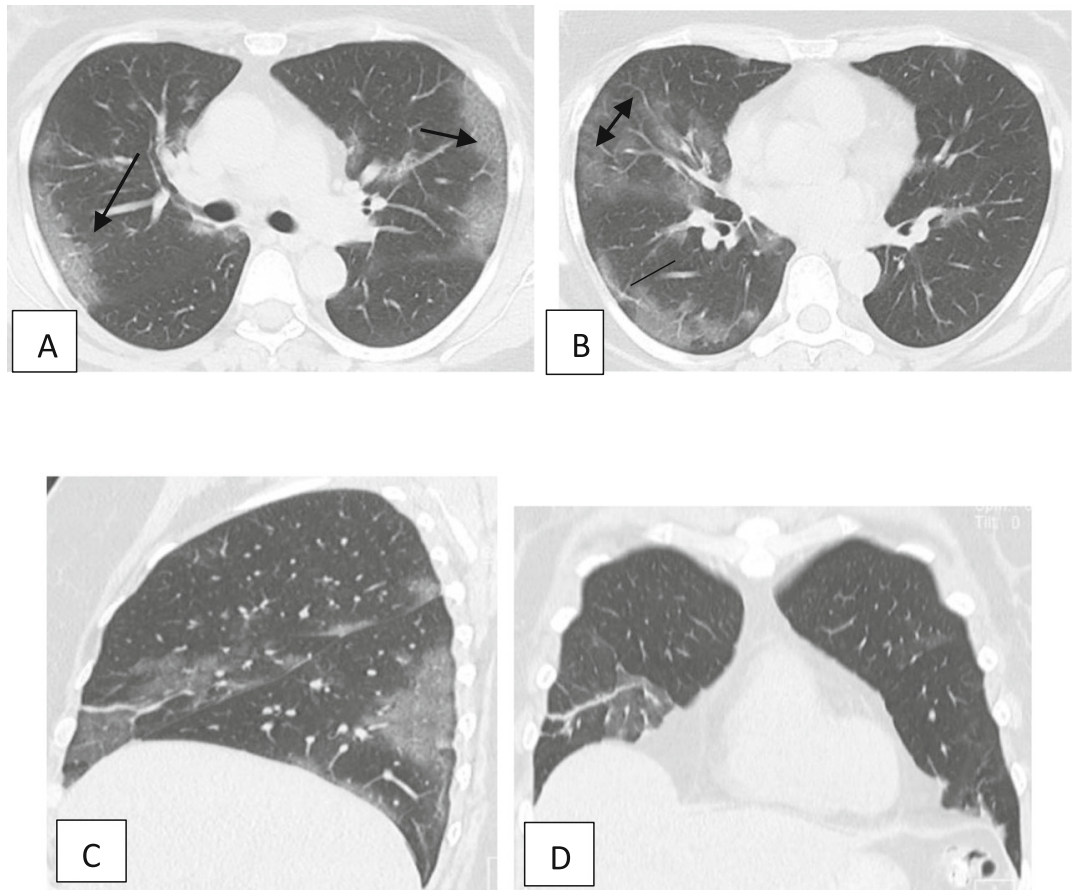

Fig. 5 Unenhanced chest CT of a 43-year-old female patient with COVID-19. a, b Axial lung window images show bilateral subpleural areas of CP [single head arrows], subpleural and broncho-vascular areas of ground glassing opacity [double head arrow], and fibrosis [line]. c sagittal and d coronal reformatted. CT score is 11 

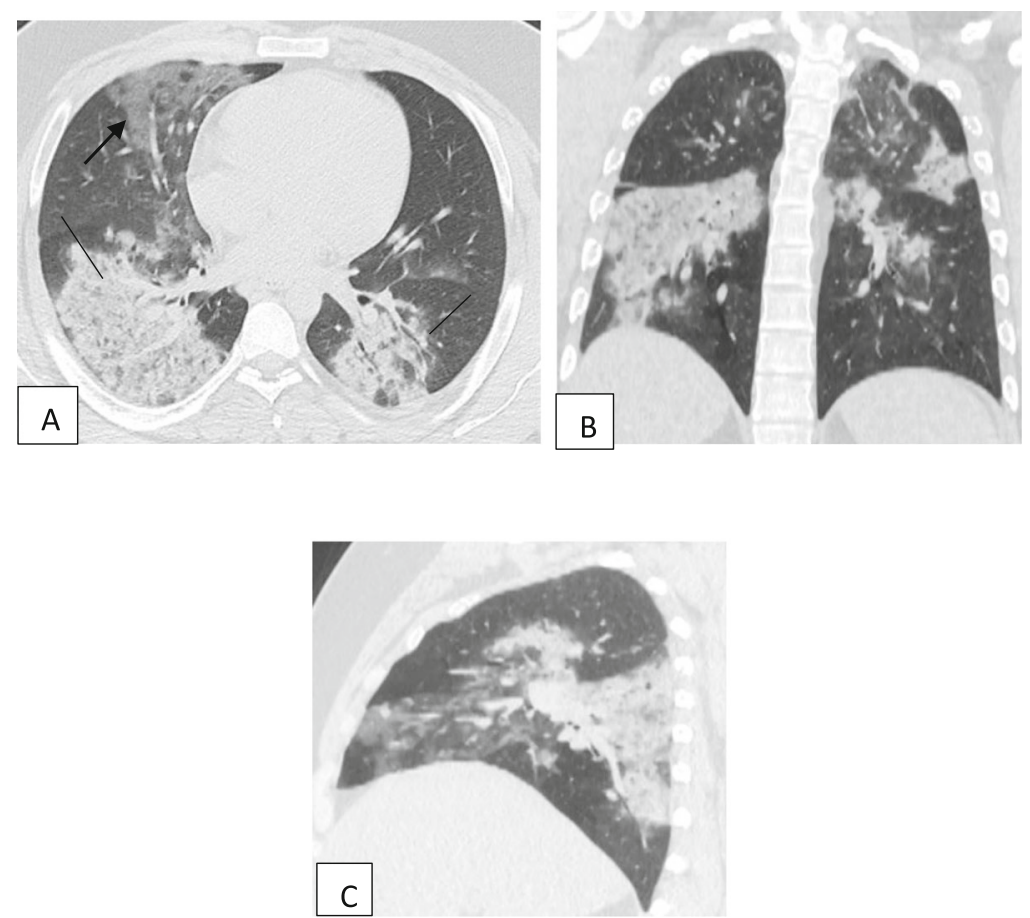

Fig. 6 Unenhanced chest CT of a 21-year-old male patient with COVID-19. a An axial lung window image shows bilateral subpleural and broncho-vascular areas of ground glassing opacity [single head arrow] and crazy paving and consolidation [line]. b Coronal and c sagittal reformatted images. CT score is 18
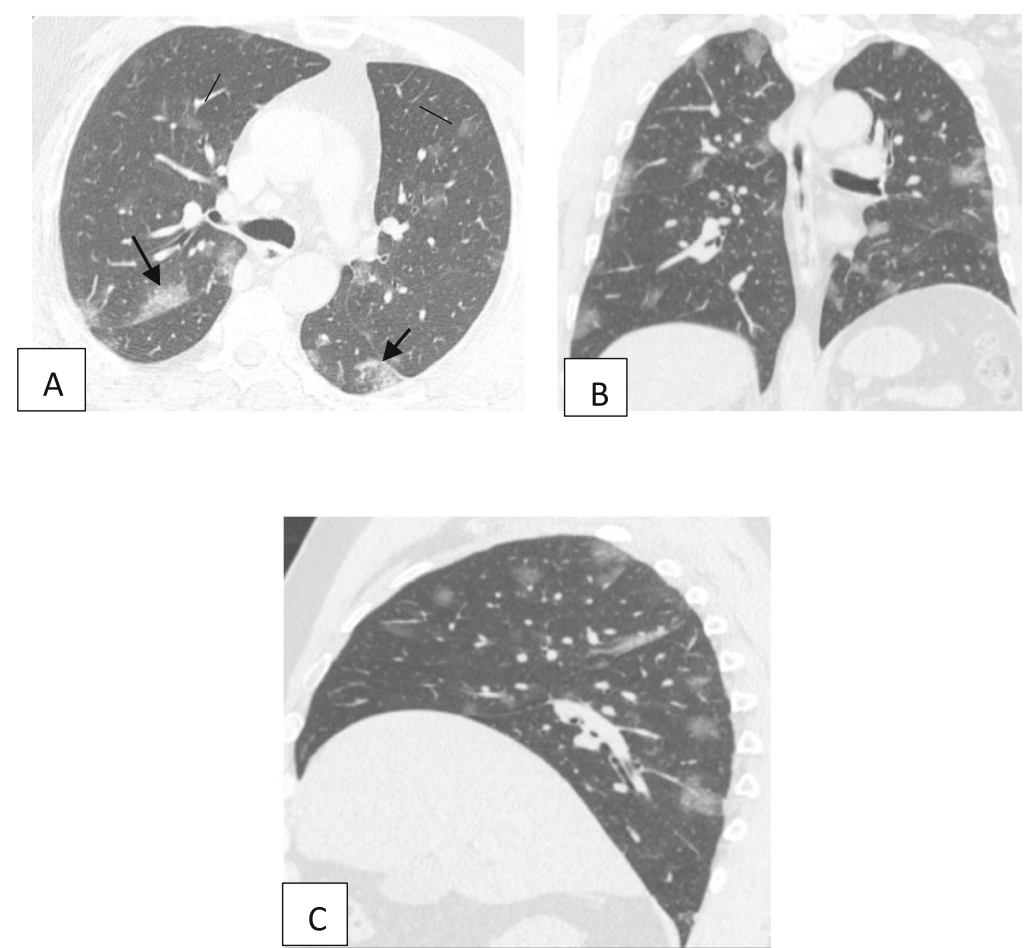

Fig. 7 Unenhanced chest CT of a 52-year-old male patient with COVID-19. a An axial lung window image shows bilateral subpleural and broncho-vascular areas of ground glassing opacity [lines] and crazy paving [arrows]. b Coronal and c sagittal reformatted images. CT score is 12 

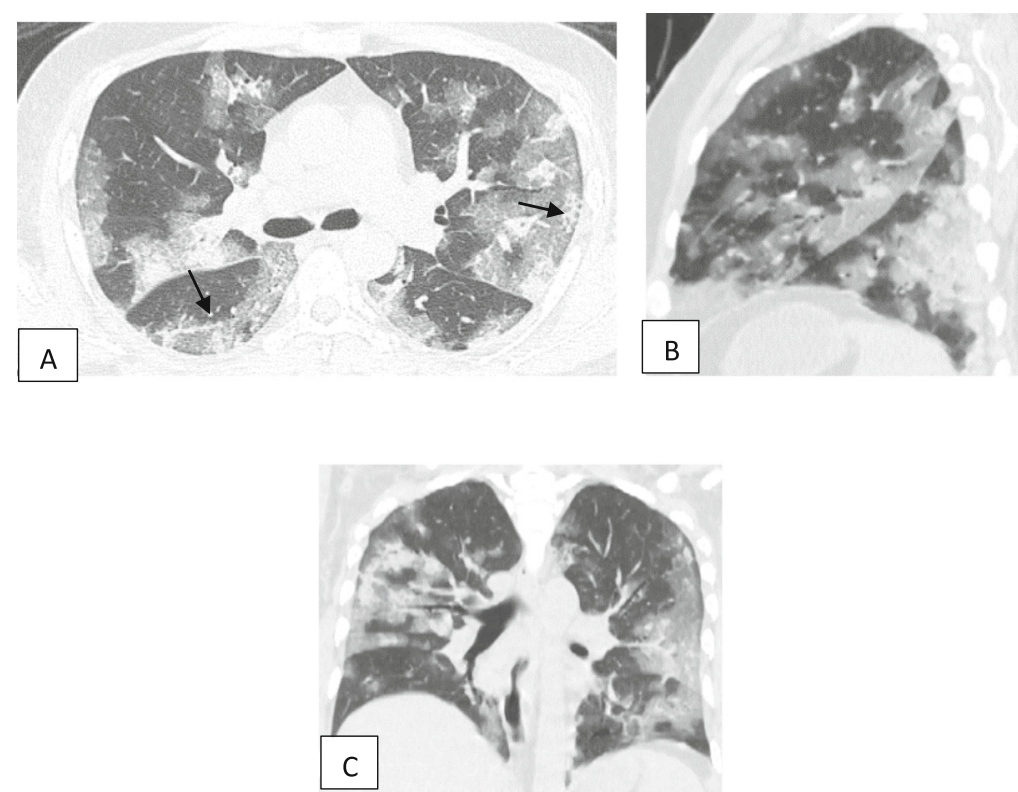

Fig. 8 Unenhanced chest $C T$ of 52-year-old male patient with COVID-19. a Axial lung window image shows bilateral subpleural and broncho-vascular areas of ground glassing opacity, consolidation, crazy paving, and fibrosis [single head arrows]. b Sagittal and c coronal reformatted images. CT score is 19
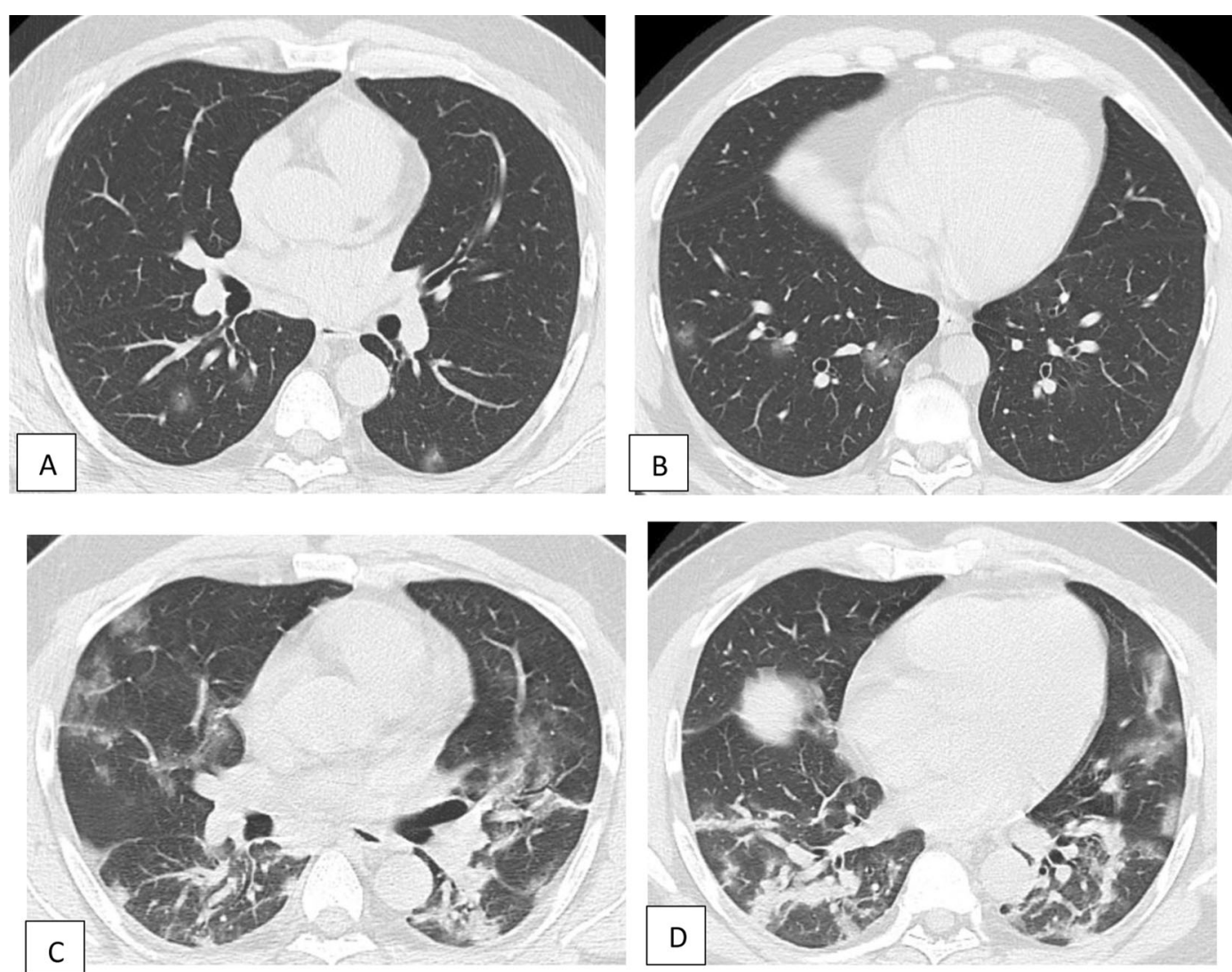

Fig. 9 Unenhanced chest CT of a 42-year-old male patient with COVID-19. a, b Axial lung window images showing subpleural and bronchovascular small areas of GGO with CT score 5. $\mathbf{c}$, $\mathbf{d}$ Axial lung window images after 5 days showing the progression of the disease with increase in the number and size of the GGO areas with the development of frank areas of consolidation and fibrosis with CT score of 17 


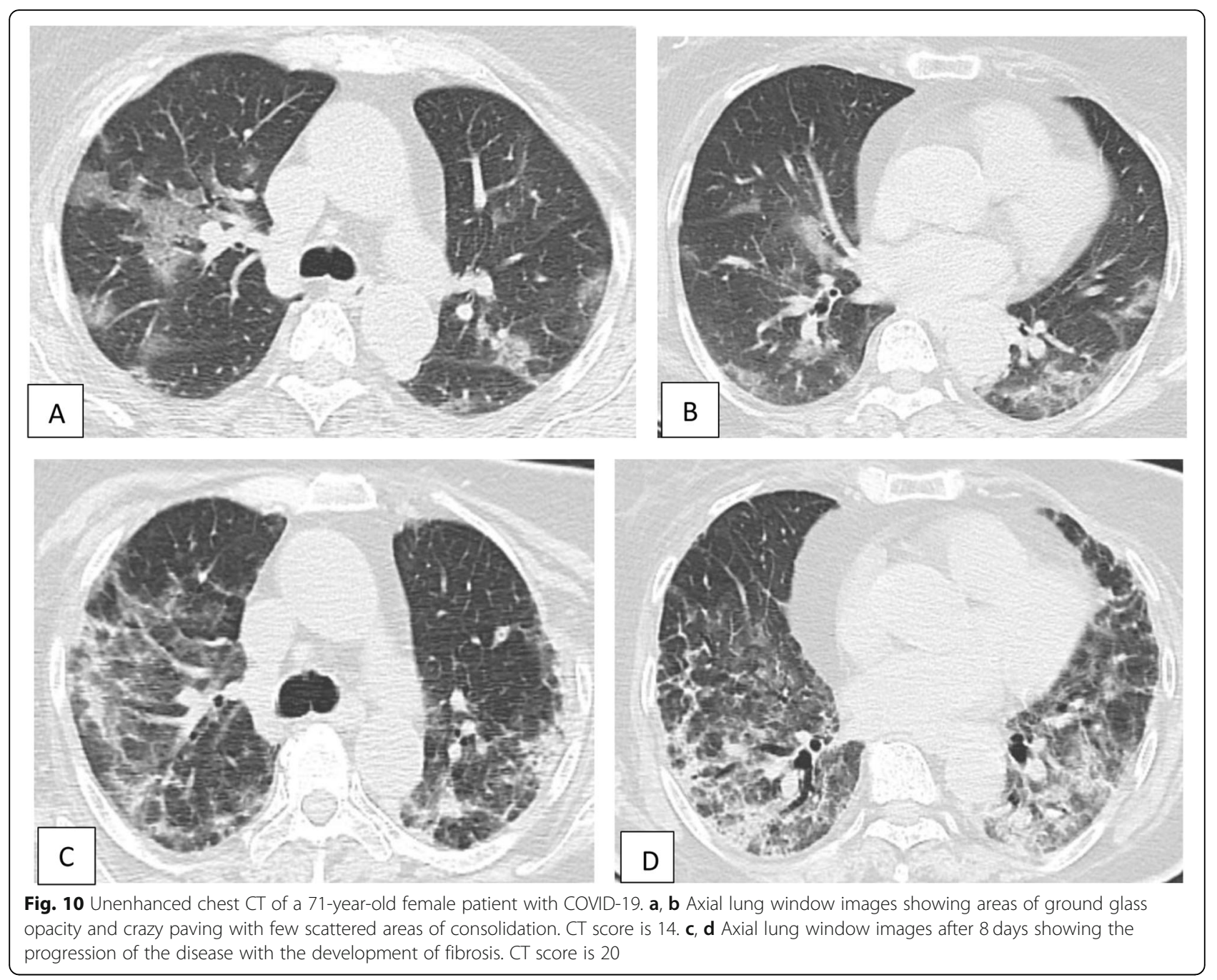

Regarding laterality of positive chest CT cases, bilateral affection was $88 \%$ while unilaterality was only $12 \%$ [7.5\% right lung and 4.5\% left lung] (Figs. 1 and 2). Regarding the distribution of lesions, both peripheral and central were the highest percent $67 \%$ while peripheral distribution alone was $31 \%$ (Fig. 1) and central was only $2 \%$ (Fig. 2).

Regarding the pattern of the lesions, GGO was the highest pattern evident seen in 191/487 representing $39.2 \%$, after which came consolidation in 152/487 [31.2\%], then CP in 74/487 [ 15.2\%] (Fig. 4), after which came fibrosis in 67/487 [13.7\%] (Fig. 5), with lymph nodes seen only in three cases representing the least pattern giving only $0.6 \%$ (Table 5 ).

There was a high statistical significance between both elevated serum ferritin and serum LDH and CT staging using the Kruskal-Wallis test where ferritin was increased in $18.4 \%$ in the mild stage, $63 \%$ in the moderate, and $100 \%$ in the severe stage (Table 6) while LDH was increased in $39.4 \%, 92.3 \%$, and $100 \%$ mild, moderate, and severe stages, respectively (Table 7).

Fifteen cases [6\%] were critical cases that needed ICU admission, and all were in the severe stage while all the negative chest CT patients, mild, moderate, and the rest

Table 2 Gender comparison between different CT stages

\begin{tabular}{lllll}
\hline & Mild & Moderate & Severe & Total \\
\hline Female & $53,46.4 \%$ & $23,35.4 \%$ & $3,14.3 \%$ & 79 \\
Male & $61,53.5 \%$ & $42,64.6 \%$ & $18,85.7 \%$ & 121 \\
Total & $114,100 \%$ & $65,100 \%$ & $21,100 \%$ & $200,100 \%$ \\
\hline
\end{tabular}


Table 3 Age comparison between different CT stages

\begin{tabular}{lllll}
\hline & Mild & Moderate & Severe & Total \\
\hline $\mathbf{1 7 - 3 0}$ & $27,23.7 \%$ & $9,13.8 \%$ & $0,0 \%$ & 36 \\
$\mathbf{3 1 - 5 0}$ & $47,41.2 \%$ & $24,36.9 \%$ & $10,47.6 \%$ & 81 \\
$\mathbf{5 1 - 8 2}$ & $40,35.1 \%$ & $32,49.2 \%$ & $11,52.4 \%$ & 21 \\
Total & 114 & 65 & 21 & 200 \\
\hline
\end{tabular}

of the severe CT score patients 235/250 [94\%] either took treatment in the ward or sent for home quarantine (Table 8).

Regarding ICU patients, all showed severe CT scoring ranging from 21 to 23; they included one female [66 age with diabetes mellitus] and 14 male cases, with three cases that were in the age group of 31 till 50 years [one case with no comorbidity, one case with hypertension, and one with diabetes mellitus] and 11 cases that were in the age group of 51 till 82, all with comorbidity. All the patients showed bilateral lung involvement along with peripheral and central distribution. All the ICU patients showed very high levels of serum ferritin [75 percentile] reaching up to 1264 , more than three times the normal range, and serum LDH [75 percentile] reaching up to 901 , more than four times the normal range.

A follow-up chest CT, serum ferritin, and serum LDH were done for 8 cases [3.2\%] in the interval of 3-10 days from the first chest CT due to deterioration of the clinical picture; their data were as follows: 4 cases of mild category, three of which became moderate and one remained mild but with a higher score; 3 moderate cases became severe; and one severe case remained in the severe category but with a higher score. All showed an increase in both serum ferritin and LDH compared to the first result (Table 9).

\section{Discussion}

COVID-19 is a new highly contagious infectious disease that causes lower respiratory inflammation; it starts by alveolar hyaline membrane injury or interstitial edema which appears on CT as GGO then progresses to alveolar filling with exudate which is the consolidation after which an autoimmune reaction begins by activation of both humeral and cellular immunity mediated by virus- specific $B$ and $T$ cells causing the formation of proinflammatory cytokines which are the cause of interlobular septal thickening, giving rise to first crazy paving then lung fibrosis [12].

The clinical picture of the disease is diverse ranging from asymptomatic or subclinical forms to critically ill patients with acute respiratory distress syndrome [ARDS] or multi-organ failure which can occur as short as 9 days from the onset of symptoms [9], so not only diagnosis of the disease is mandatory but also monitoring the clinical course of the disease is of paramount importance to triage patients who are in need of urgent intensive medical care. Extension of pulmonary affection is easily assessed by chest CT but this is not suitable for critically ill patients, so a laboratory biomarker of disease progression is needed and here comes the role of LDH and ferritin.

LDH is an enzyme that converts lactate to pyruvate in the process of tissue breaking down, so patients with severe COVID-19 pneumonia shows an increase in the serum LDH [13]; also, ferritin is increased because it is secreted either by the macrophages that produce cytokines and account for the main immune cells present in lung parenchyma or by cytokines themselves such as interleukin-6 [14].

From 250 cases with confirmed RT-PCR for COVID19, 185 [74\%] patients were symptomatizing from which 22/185 [11.2\%] showed normal chest CT; this was in agreement with Yang and Yan [15] who reported 11.8\% [symptomatizing patient showing normal chest CT] while Bernheim et al. [16] showed 56\%; this big difference in the percentage between the current study/Yang and Yan [15] and Bernheim et al. [16] could be explained that the latter study was done only within the first 2 days from onset of symptoms in contrary to our study and Yang and Yan that were done within 1 week.

Table 4 Comparison between age and different CT stages [Kruskal-Wallis test]

\begin{tabular}{llllll}
\hline & Number & Median & H & Sig \\
\hline Normal & 50 & 39 & & \\
Mild & 114 & 42 & & \\
Moderate & 65 & 50 & & \\
Severe & 21 & 50 & & \\
Total & 250 & & 20.91 & HS \\
& & & & \\
\hline
\end{tabular}


Table 5 Pattern of CT chest pulmonary manifestations in different CT stages

\begin{tabular}{lllll}
\hline & Mild & Moderate & Severe & Total \\
\hline GGO & $105 / 114,92.1 \%$ & $65 / 65,100 \%$ & $21 / 21,100 \%$ & $191,39.2 \%$ \\
Consolidation & $81 / 114,71 \%$ & $53 / 65,81.5 \%$ & $18 / 21,85.7 \%$ & $152,31.2 \%$ \\
CP & $22 / 114,19.2 \%$ & $39 / 65,60 \%$ & $13 / 21,61.9 \%$ & $74,15.2 \%$ \\
Fibrosis & $21 / 114,16.4 \%$ & $25 / 65,60 \%$ & $21 / 21,100 \%$ & $67,13.7 \%$ \\
Lymph node & $0 / 114,0 \%$ & $3 / 65,4.6 \%$ & $0 / 21,0 \%$ & $3,0.6 \%$ \\
Total & & & & $487,100 \%$ \\
\hline
\end{tabular}

Thirty-seven [14.8\%] patients were asymptomatic and showed positive chest CT findings; this clinicalradiological discrepancy was evident in previous studies like Chung et al. [17] which showed $14 \%$ while Inui et al. [18] which reported 39.2\%; this was explained by a lot of theories, like those patients were exposed to COVID-19 virus before and hence developed immunity leading to a subclinical presentation, or they are having a recent infection and in the recovery period with subsiding of symptoms at the time of chest CT done but an absence of clear clinical history of infection and lacking CT features of healing COVID-19 pneumonia weaken this theory, and the only logical explanation is that there is a time interval between chest CT manifestation and developing symptoms with the former begins first but this hypothesis needs to be more investigated [17, 18]; unfortunately, in the current study, none of those patients was followed up to see if they have developed symptoms or not.

Regarding the comparison between gender and different stages of COVID-19 pulmonary affection, there was no gender difference in the mild stage while in the moderate stage males represented $64.6 \%$ almost double of the females who were $35.4 \%$ while in the severe stage males were $85 \%$ compared to only $14.3 \%$ for females; this result was in concordance with Jin et al. [19] and Hiroki et al. [20] with the latter stating that male gender may be a predictor of a more severe form of COVID-19.

Regarding the age, there was a statistically significant correlation between older age and severity of chest CT manifestations as no patient in the severe stage was under 30 years while there was no difference between 31 and 50 years group and 51-82 years group showing $47.6 \%$ and $52.4 \%$, respectively; this was in agreement with the US Centers for Disease Control and Prevention reporting that 8 in 10 COVID-19-related deaths have been among people aged 65 years or over [21].

Regarding the distribution of lung manifestations, unilateral lung affection was only observed in $24 / 200$ [12\%] while bilaterally was seen in $176 / 200$ [88\%], and that was in agreement with almost all previous publications [7-9, $16,17]$.

Peripheral involvement was seen in 62/200 [31\%] and broncho-vascular in $4 / 200$ [2\%], while both peripheral and broncho-vascular involvement was the highest percentage being 134/200 [67\%]; this finding was in agreement with Wang et al. [8] who reported $56.4 \%$ both peripheral and broncho-vascular distribution. On the contrary, Shi et al. [9] reported more percentage of peripheral distribution 54\% compared to $44 \%$ diffuse, and this can be explained that this study included a small number of patients, only 81 patients with some of which were asymptomatic.

Regarding the pattern of chest CT pulmonary affection in different stages, GGO was the highest percentage accounting for $191 / 487$ [39.2\%], followed by consolidation $152 / 487$ [31.2\%], then fibrosis $74 / 487$ [15.2\%], and CP $67 / 487$ [13.7\%], with lymph node only seen in $3 / 487$ [0.6\%]; these findings were in agreement with Song et al. [22].

Since the outbreak of the COVID-19 pandemic, many studies showed that the inflammatory cytokine storm is the main cause of complication of COVID-19 pneumonia leading to acute respiratory stress syndrome and multi-organ failure and even death, so laboratory detection of increased serum inflammatory markers is mandatory in order to provide those patients early proper treatment [23].

In the current study, there was a statistically high significance between increased levels of both serum ferritin

Table 6 Comparison between elevated serum ferritin in different CT stages [normal value is between 30 and $400 \mathrm{ng} / \mathrm{mL}$ ] [KruskalWallis test]

\begin{tabular}{lllllll}
\hline & Number & Median & 25Perc. & 75Perc. & H & Sig. \\
\hline Mild & $21 / 114,18.4 \%$ & 492 & 480 & 557.5 & & \\
Moderate & $41 / 65,63 \%$ & 634 & 518.5 & 765 & & \\
Severe & $21 / 21,100 \%$ & 880 & 772 & & & 27.451 \\
Total & $83 / 200,41.5 \%$ & & & & HS \\
\hline
\end{tabular}


Table 7 Comparison between elevated serum LDH in different CT stages [normal value is between 135 and $225 \mathrm{U} / \mathrm{L}$ ] [Kruskal-Wallis test]

\begin{tabular}{lllllll}
\hline & Number & Median & 25Perc. & 75Perc. & H & Sig. \\
\hline Mild & $45 / 114,39.5 \%$ & 340 & 237 & 418 & & \\
Moderate & $60 / 65,92.3 \%$ & 361.5 & 300 & 450 & & \\
Severe & $21 / 21,100 \%$ & 758 & 621.5 & 901 & & \\
Total & $126 / 200,63 \%$ & & & & 51.933 & HS \\
\hline
\end{tabular}

and LDH in correlation with CT staging, where ferritin was increased in $18.4 \%$ in the mild stage, $63 \%$ in the moderate, and $100 \%$ in the severe stage; these findings were in agreement with Lin et al. [24] who reported that patients with severe COVID-19 pneumonia showed higher levels of serum ferritin than the non-severe patients using a multivariate logistic regression analysis showing that the serum ferritin level was an independent risk factor for disease severity.

LDH was increased in $39.4 \%$, 92.3\%, and $100 \%$ mild, moderate, and severe stages, respectively, and this was in concordance with $\mathrm{Wu}$ et al. [13] who reported that the higher LDH levels were found in patients with severe COVID-19. All the ICU patients showed a marked increase reaching up to more than four times the normal; these results were in agreement with the pooled study done by Henry et al. [25] who stated that increased LDH was associated with significantly increased odds of severe COVID-19 in both case-control studies and retrospective studies.

In spite that CT scoring is a subjective method for assessing the severity of COVID-19 pneumonia, it correlates well with the clinical manifestations and laboratory findings of the disease [26] helping to triage patients and reserving health care resources so only the ones with severe lung affection could be closely monitored in order to provide early aggressive treatment; in the current study, comparing the CT score for patients admitted to ICU and patients who were treated either in the hospital or at home, all the patients in ICU were in severe stage with CT scores ranging from 21 to 23 , and this was in agreement with the study of 236 patients done by Colombi et al. [27] who reported a positive correlation between the extent of CT lung manifestations and intensive care unit admission or death. Also,

Table 8 Comparison between ICU patients and ward/home patients in different $C T$ stages

\begin{tabular}{llllll}
\hline & Negative chest CT & Mild & Moderate & Severe & Total \\
\hline Ward/home & 50 & 114 & 65 & 6 & $235,94 \%$ \\
ICU & 0 & 0 & 0 & 15 & $15,6 \%$ \\
Total & 50 & 114 & 65 & 21 & 250 \\
\hline
\end{tabular}

Francone et al. [25] reported that CT score was significantly higher in critical COVID-19 patients ranging from 15 to 24 with $20.3 \pm 3$ [mean value $\pm \mathrm{SD}$ ] than in the mild stage range $0-19$ with $8.7 \pm 4$ [mean value $\pm \mathrm{SD}$ ].

This study has limitations as none of the patients was in the pediatric age group; the study included only patients within the first week of onset of symptoms or positive RT-PCR, so late chest manifestations of the disease could not be investigated; also, there was no followup of cases that were asymptomatic with positive chest CT findings to find out if they developed symptoms or not or the patients that were symptomatic and with negative chest CT findings to see if they developed imaging manifestations or not, but this could be explained that the time of the study was at the peak period of the disease with a large number of cases and limited health care resources.

\section{Conclusion}

Chest CT findings are crucial for early diagnosis of COVID-19 disease especially for asymptomatic patients for early quarantine aiming to decrease the spread of the disease. Old age and male sex are considered risk factors for poor prognosis. CT score, serum ferritin, and LDH play an important role in predicting the short-term outcome of the patients allowing prompt effective clinical monitoring and intervention to decrease both morbidity and mortality.

Table 9 Follow-up cases

\begin{tabular}{llllll}
\hline $\begin{array}{l}\text { 1st CT } \\
\text { score }\end{array}$ & $\begin{array}{l}\text { 2nd CT } \\
\text { score }\end{array}$ & $\begin{array}{l}\text { First } \\
\text { ferritin }\end{array}$ & $\begin{array}{l}\text { Second } \\
\text { ferritin }\end{array}$ & $\begin{array}{l}\text { First } \\
\text { LDH }\end{array}$ & $\begin{array}{l}\text { Second } \\
\text { LDH }\end{array}$ \\
\hline 7 & 13 & Normal & 656 & 270 & 319 \\
5 & 15 & Normal & 416 & Normal & 298 \\
6 & 11 & Normal & 432 & Normal & 282 \\
7 & 8 & 480 & 490 & Normal & 230 \\
13 & 23 & 651 & 1232 & 360 & 620 \\
14 & 20 & 460 & 580 & 232 & 742 \\
14 & 21 & 455 & 232 & 733 & 505 \\
20 & 23 & 532 & 1180 & 501 & 800 \\
\hline
\end{tabular}




\section{Abbreviations}

RT-PCR: Real-time reverse transcriptase-polymerase chain reaction;

LDH: Lactate dehydrogenase; GGO: Ground glass opacity; CP: Crazy paving

\section{Acknowledgements}

Not applicable.

\section{Authors' contributions}

All authors have read and approved the manuscript. Dr. RB, the corresponding author: Idea. Reviewing chest CT findings. Writing the manuscript. Dr. AS: Collecting data from the hospital information system. Helping in writing the manuscript

\section{Funding}

Not applicable as no funding was received for this study.

\section{Availability of data and materials}

All the data are available at the Ain-Shams University hospital information system [HIS]

\section{Declarations}

\section{Ethics approval and consent to participate}

This study was approved by the Faculty of Medicine Ain-Shams University with no number available.

\section{Consent for publication}

Not applicable as the manuscript has no individual person's data in any form.

\section{Competing interests}

The authors declare that they have no competing interests.

\section{Author details}

'Department of Radiodiagnosis, Faculty of Medicine, Ain Shams University, Cairo, Egypt. ${ }^{2}$ Department of Anesthesia, Intensive Care and Pain Management, Faculty of Medicine, Ain Shams University, Cairo, Egypt

Received: 4 December 2020 Accepted: 11 March 2021

Published online: 30 March 2021

\section{References}

1. Guan C, Lv Z, Yan S et al (2020) Imaging features of coronavirus disease 2019 (COVID-19): evaluation on thin-section CT. Acad Radiol 27(5):609-613. https://doi.org/10.1016/j.acra.2020.03.002

2. Hani C, Trieu NH, Saab I et al (2020) COVID-19 pneumonia: a review of typical CT findings and differential diagnosis. Diagn Intervent Imaging 101(5):263-268. https://doi.org/10.1016/j.diii.2020.03.014

3. Sultan O, Altameemi $H$, Alghazali D et al (2020) Pulmonary manifestations of COVID-19: changes within 2 weeks duration from presentation. Egypt J Radiol Nucl Med 51:105-111

4. Young D, Tatarian L, Mujtaba G et al (2020) Chest CT versus RT-PCR for diagnostic accuracy of COVID-19 detection: a meta-analysis. J Vasc Med Surg 392(3):1-4

5. Ai T, Yang Z, Hou H, Zhan C, Chen C, Lv W, Tao Q, Sun Z, Xia L (2020) Correlation of chest CT and RT-PCR testing for coronavirus disease 2019 (COVID-19) in China. A report of 1014 cases. Radiology 296(2):E32-E40. https://doi.org/10.1148/radiol.2020200642

6. He JL, Luo L, Luo ZD, Lyu JX, Ng MY, Shen XP, Wen Z (2020) Diagnostic performance between CT and initial real-time RTPCR for clinically suspected 2019 coronavirus disease (COVID-19) patients outside Wuhan, China. Respir Med 168:105980. https://doi.org/10.1016/j.rmed.2020.105980

7. Sabri Y, Nassef A, Ibrahim I et al (2020) CT chest for COVID-19, a multicenter study-experience with 220 Egyptian patients. Egypt J Radiol Nucl Med 51(1): 144. https://doi.org/10.1186/s43055-020-00263-6

8. Wang K, Kang S, Tian R et al (2020) Imaging manifestations and diagnostic value of chest CT of coronavirus disease 2019 (COVID-19) in the Xiaogan area. Clin Radiol 75(5):341-347. https://doi.org/10.1016/j.crad.2020.03.004

9. Shi H, Han X, Jiang N, Cao Y, Alwalid O, Gu J, Fan Y, Zheng C (2020) Radiological findings from 81 patients with COVID-19 pneumonia in Wuhan,
China: a descriptive study. Lancet Infect Dis 20(4):425-434. https://doi.org/1 0.1016/S1473-3099(20)30086-4

10. Hansell DM, Bankier AA, MacMahon $\mathrm{H}$ et al (2008) Fleischner Society: glossary of terms for thoracic imaging. Radiographics 246(3):697-722

11. Pan F, Ye T, Sun P, Gui S, Liang B, Li L, Zheng D, Wang J, Hesketh RL, Yang $L$, Zheng C (2020) Time course of lung changes on chest CT during recovery from 2019 novel coronavirus (COVID-19) pneumonia. Radiology 295(3):715-721. https://doi.org/10.1148/radiol.2020200370

12. Li X, Geng M, Peng Y, Meng L, Lu S (2020) Molecular immune pathogenesis and diagnosis of COVID-19. J Pharm Anal 10(2):102-108. https://doi.org/10.1 016/j.jpha.2020.03.001

13. Wu M, Yao L, Wang Y, Zhu XY, Wang XF, Tang PJ, Chen C (2020) Clinical evaluation of potential usefulness of serum lactate dehydrogenase (LDH) in 2019 novel coronavirus (COVID-19) pneumonia. Respir Res 21(1):171-177. https://doi.org/10.1186/s12931-020-01427-8

14. Gomez-Pasyora J, Weigand M, Kim J et al (2020) Hyperferritinemia in critically ill COVID-19 patients - is ferritin the product of inflammation or a pathogenic mediator? Clin Chim Acta 509:249-251. https://doi.org/10.1016/j. cca.2020.06.033

15. Yang W, Yan F (2020) Patients with RT-PCR confirmed COVID-19 and normal chest CT. Radiology 2020:200702

16. Bernheim A, Mei X, Huang M et al (2020) Chest CT findings in coronavirus disease-19 (COVID-19): relationship to duration of infection. Radiology 2020: 200463

17. Chung M, Bernheim A, Mei X et al (2020) CT imaging features of 2019 novel coronavirus (2019-nCoV). Radiology. 2020:200230

18. Inui S, Fujikawa A, Jitsu M, Kunishima N, Watanabe S, Suzuki Y, Umeda S, Uwabe Y (2020) Chest CT findings in cases from the cruise ship "Diamond Princess" with coronavirus disease 2019 (COVID-19). Radiology 17(2):2020. https://doi.org/10.1148/ryct.2020200110

19. Jin JM, Bai P, He W et al (2020) Gender differences in patients with COVID19: focus on severity and mortality. Public Health 29:2020. https://doi.org/1 0.3389/fpubh.2020.00152

20. Hiroki U, Toshiki K, Hisato T et al (2020) Gender difference is associated with severity of coronavirus disease 2019 infection: an insight from a metaanalysis. Crit Care Explorations 2:148-153

21. Mahase E (2020) Covid-19: Why are age and obesity risk factors for serious disease? BMJ. https://doi.org/10.1136/bmj.m4130

22. Song F, Shi N, Shan F et al (2020) Emerging coronavirus 2019-nCOV pneumonia. Radiology 295:2020200274

23. Chen G, Wu D, Guo W, Cao Y, Huang D, Wang H, Wang T, Zhang X, Chen $H$, Yu H, Zhang X, Zhang M, Wu S, Song J, Chen T, Han M, Li S, Luo X, Zhao J, Ning Q (2020) Clinical and immunological features of severe and moderate coronavirus disease 2019. J Clin Invest 130(5):2620-2262. https:// doi.org/10.1172/JCl137244

24. Lin Z, Long F, Chen $X$ et al (2020) Serum ferritin as an independent risk factor for severity in COVID-19 patients. J Inf Secur 81(4):647-679. https:// doi.org/10.1016/j.jinf.2020.06.053

25. Henry BM, Aggarwal G, Wong J, Benoit S, Vikse J, Plebani M, Lippi G (2020) Lactate dehydrogenase levels predict coronavirus disease 2019 (COVID-19) severity and mortality: a pooled analysis. Am J Emerg Med 38(9):1722-1726. https://doi.org/10.1016/j.ajem.2020.05.073

26. Francone M, lafrate F, Masci G et al (2020) Chest CT score in COVID-19 patients: correlation with disease severity and short-term prognosis. Eur Radiol 30(12):6808-6817. https://doi.org/10.1007/s00330-020-07033-y

27. Colombi D, Bodini FC, Petrini M et al (2020) Well-aerated lung on admitting chest CT to predict adverse outcome in COVID-19 pneumonia. Radiology 296(2):E86. https://doi.org/10.1148/radiol.2020201433

\section{Publisher's Note}

Springer Nature remains neutral with regard to jurisdictional claims in published maps and institutional affiliations. 